\title{
A Simple Stochastic Differential Equation with Discontinuous Drift
}

\author{
Maria Simonsen, John Leth, Henrik Schioler \\ Dept. of Electronic Systems, Automation and Control \\ University of Aalborg, Denmark \\ $\{\mathrm{msi}, \mathrm{jjl}$, henrik\}@es.aau.dk
}

\author{
Horia Cornean \\ Dept. of Mathematical Sciences \\ University of Aalborg, Denmark \\ cornean@math.aau.dk
}

\begin{abstract}
In this paper we study solutions to stochastic differential equations (SDEs) with discontinuous drift. We apply two approaches: The Euler-Maruyama method and the Fokker-Planck equation and show that a candidate density function based on the Euler-Maruyama method approximates a candidate density function based on the stationary Fokker-Planck equation. Furthermore, we introduce a smooth function which approximates the discontinuous drift and apply the Euler-Maruyama method and the Fokker-Planck equation with this input. The point of departure for this work is a particular SDE with discontinuous drift.
\end{abstract}

\section{Introduction}

Since the pioneering work by Einstein on Brownian motion [3], stochastic differential equations (SDEs) have been intensively studied, with the foundation for SDEs developed by Ito and Stratonovich, e.g. see [7, 17] and references therein. Well-developed theories for various sub-disciplines of SDEs have been developed ever since, such as stability- and control theory [8, 11, 10]. Lately, SDEs have been used in the generalization of hybrid dynamical systems [2, 6, 13]. Moreover, today a variety of applications and numerical methods exist for SDEs, see [15, 9] and references therein.

Comment to the above theories is that they are all developed under (weak) regularity conditions on the drift and diffusion coefficients. Such conditions are, of course, necessary in order to develop an applicable/operational theory. However, it is also of interest to study special cases (or classes) where the standard regularity conditions fails but fundamental properties of the SDEs are still valid such as existence and uniqueness of solutions. As an example, we recall that a necessary condition for existence of solutions for an (deterministic) ordinary differential equation (ODE), $\dot{x}=f(x)$, is that the right-hand side of the ODE, that is the vector field $f$, should be continuous in the state variable $x$. However, the Cauchy problem $\dot{x}=\operatorname{sign}(x), x(0)=x_{0}$, with sign denoting the sign-function, has a solution for all initial values $x_{0}$, despite the fact that sign is discontinuous at zero (we remark that $\operatorname{sign}(0)=0$ by definition, if this was not the case no solution would exist at $x_{0}=0$ ). As discontinuous functions often appear in applications, ODEs such as $\dot{x}=\operatorname{sign}(x)$ should and have been studied, see [4] and references therein.

In this work in progress we initiate a study of SDEs with discontinuous drift. This should be seen as a part of a larger scope with focuses on the intersection between SDEs and switching dynamics, a field only scarcely explored so far, see [12] and references therein for related work. Within this larger scope theoretical questions to be answered are how to define solutions to SDEs with state dependent switching (in particular with discontinuous drift), when solutions cannot be patched together of segments of positive time duration and can any applicable results be obtained by applying the Euler Maruyama method [14, 5], which is a simple time discrete approximations technique, to SDEs whose drift do not meet the regularity conditions? Here, we exemplify these problems by studying solution candidates to a 
particular SDE having discontinuous drift coefficient. More precisely, we consider the SDE

$$
d x_{t}=-k \operatorname{sign}\left(x_{t}\right) d t+d B_{t},
$$

with $k$ a control gain, and ask how a solution can be defined.

The systems treated in [2] are assumed to have non-zeno execution in finite time. A local behavior of the Wiener process $B_{t}$ is that in every time interval of the form $[0, \varepsilon)$, with $\varepsilon>0, B_{t}$ has infinitely many zeros, that is, the process which fulfills

$$
d x_{t}=d B_{t}
$$

crosses zero infinitely many times, see [1]. In (1) the process $x_{t}$ is forced to proceed against zero, so we conjecture that the solution to (1) (if it exists) also crosses zero infinitely many times in finite time.

We use several methods in the attempt to give a meaningful/operational definition of solutions to (1) based on density functions and their probabilistic properties. We start by using the Euler-Maruyama method to approximate numerical solutions to (1), which values are presented in histograms for particular time instant. These histograms can be considered as an approximation to the density function for solutions to (1). We investigate the influence of the step-size and the control gain in the simulations. Furthermore, from the Euler-Maruyama method we obtain recursively defined density functions which, under stationary conditions, appears to converge to the outcome of the second approach which departure from the Fokker-Planck equation. It is interesting to note that the candidate density function obtained from the Fokker-Planck equation is derived under the assumption of stationarity while the recursive approach has no such assumptions. More precise, we obtain formulas which strongly indicate that if such a stationary density function exists then it solves the stationary Fokker-Planck equation.

As a third method, we introduce an approximation to the sign-function and apply both the EulerMaruyama method and the Fokker-Planck equation to this. A comparison with the stationary density function which solves the Fokker-Planck equation is made.

Finally, we briefly mention one approach which relate to the Euler-Maruyama method. Even though this intuitively should produce some information, we have so far not been able to obtain any meaningful results based on this method. It is included since it is believed that it does in fact carry important information.

It is important to emphasize that the presented material is work in progress and that the heuristic presented here is an initial attempt to define meaningful candidate density functions to solutions to a particular SDE with discontinuous drift. It is clear that for future work the presented material have to be set in a formal mathematical frame including proofs which validate the various procedure used to obtain candidate density functions.

\subsection{A Stochastic Differential Equation}

A general one dimensional SDE is given by

$$
d x_{t}=b\left(t, x_{t}\right) d t+\sigma\left(t, x_{t}\right) d B_{t}, \quad x_{0}=c,
$$

where $x=x_{t}$ is an $\mathbb{R}$-valued stochastic process : $[0, T] \rightarrow \mathbb{R}, b, \sigma:[0, T] \times \mathbb{R} \rightarrow \mathbb{R}$ are the drift and diffusion coefficient of $x, B=B_{t}$ is an $\mathbb{R}$-valued Wiener process, and $c$ is a random variable independent of $B_{t}-B_{0}$ for $t \geq 0$. On $[0, T]$, existence and uniqueness of a solution $x_{t}$, continuous with probability 1 , to (2) is guaranteed whenever the drift $b$ and diffusion $\sigma$ are measurable functions satisfying a Lipschitz condition together with a growth bound, both uniformly in $t$ [15, Theorem 5.2.1]. 
This paper focuses on the special case for (2), where $\sigma\left(t, x_{t}\right)=1$ and $b\left(t, x_{t}\right)=-k \operatorname{sign}\left(x_{t}\right)$ with $k>0$ a control gain and the sign-function defined by

$$
\operatorname{sign}(x)=\left\{\begin{array}{ccc}
-1 & \text { if } & x<0 \\
0 & \text { if } & x=0 \\
1 & \text { if } & x>0
\end{array} .\right.
$$

Thus, we consider the SDE

$$
d x_{t}=-k \operatorname{sign}\left(x_{t}\right) d t+d B, \quad x_{0}=c .
$$

with $c$ given.

In the next section, the Euler-Maruyama method is applied to approximate solutions to (4) and to investigate a theoretical methods to obtain candidate density functions for solutions to (4).

\section{The Euler-Maruyama Method}

The Euler-Maruyama method is a simple time discrete approximation technique which is used to approximate solutions to SDEs of the type given in (2), by discretizing the time interval $[0, T]$ in steps $0<t_{1}<\cdots<t_{n}<t_{n+1} \cdots<t_{N}$ with $N=\left\lceil\frac{T}{h}\right\rceil$, where $h=t_{n+1}-t_{n}$ is the step-length. Each recursive step is determined via the following method,

$$
x_{n+1}=x_{n}+h b\left(t_{n}, x_{n}\right)+\sigma\left(t_{n}, x_{n}\right) W_{n},
$$

where $x_{t_{i}}=x_{i}$ and $W_{n}=B_{t_{n+1}}-B_{t_{n}}$ is i.i.d. normal with mean zero and variance $h$, which we denote by $W_{n} \sim N(0, h)$.

Given an initial condition $x_{0}=c$, it is possible from (5) to approximate a solution to (2) by determination of $x_{1}, x_{2}, \ldots, x_{N}$. If the drift and diffusion coefficient in (2) are measurable, satisfy a Lipschitz condition and a growth bound, the Euler Maruyama method guarantee strong convergence to the solution of (2), [9, Theorem 9.6.2]. Hence for SDEs with discontinuous drift we can, in general, not expect the Euler Maruyama method to produce meaningful results. Nevertheless, we will in the sequel apply this method to the special case (4) in order to obtain candidate solutions.

\subsection{Analysis of the Deterministic Step}

Application of the Euler-Maruyama method to the SDE in (4) gives the recursive step

$$
x_{n+1}=x_{n}-h k \operatorname{sign}\left(x_{n}\right)+W_{n} .
$$

Now, if $x_{n}>0$, we have

$$
x_{n+1}=x_{n}-h k+W_{n} .
$$

Since $W_{n} \sim N(0, h)$, the expectation is that $x_{n+1} \in\left[x_{n}-h(k+1), x_{n}-h(k-1)\right]$ in most of the simulations. From this, we expect after a finite time $0<t<\infty$ that there exists $N \in \mathbb{N}$ such that $x_{n+N} \leq 0$. Similar result is obtained if $x_{m}<0$, then we expect that there exists $M \in \mathbb{N}$ such that $x_{m+M} \geq 0$. The influence from the control gain $k$ determines how quick the evolution of the sequence $\left\{x_{n}\right\}_{n \geq 0}$ switches around zero. In other words, a big $k$ minimizes the influence of the random variable $W_{n}$.

The Euler-Maruyama method is easy to implement in software, so following we have applied Matlab to simulate solutions to (4). 


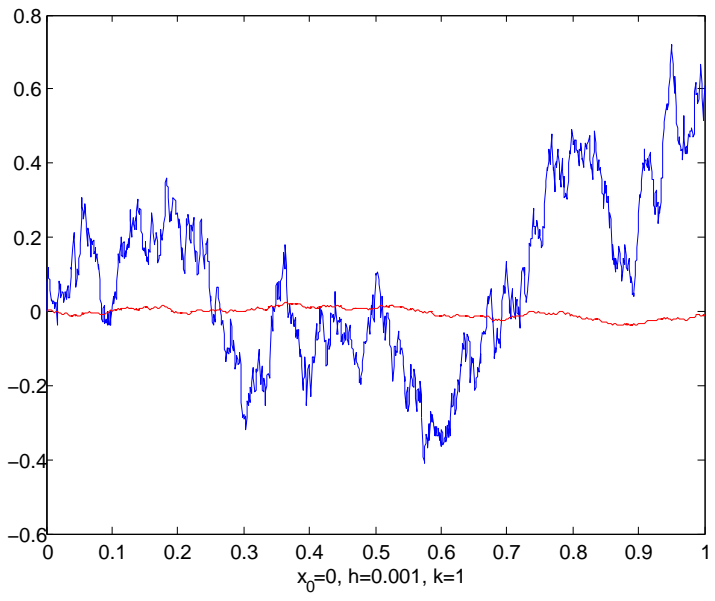

Figure 1: The blue graph shows one realization out of 500 simulations while the red graph represents the average of $x_{t}$ for $0 \leq t \leq T$

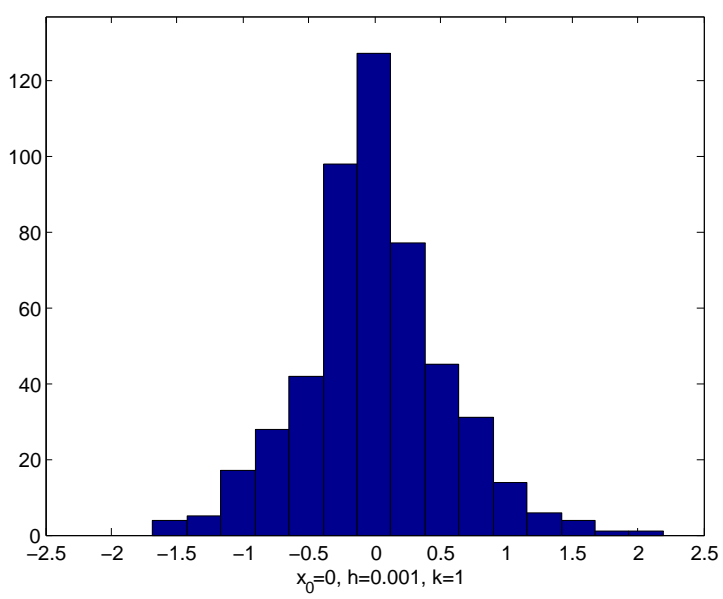

Figure 2: Resulting histogram of 500 simulations of $x_{T}$.

\subsection{Numerical solutions to a SDE with Discontinuous Drift}

We consider the recursive step in (6) and simulate the evolution of the stochastic process. For all simulations the initial condition is chosen to be $x_{0}=0$ and the considered time interval is $[0, T]$ where $T=1$. The step-length is $h$ such that the number of simulated steps is $N=\left\lceil\frac{T}{h}\right\rceil$. All simulations are repeated 500 times and histograms of the resulting values of $x_{T}$ are presented.

In figure 11, one realization of a solution to the SDE in (4) is shown together with the average values of all the 500 simulations in the time interval $[0, T]$. The average of $x_{t}$ is close to zero for all $0 \leq t \leq T$.

Figure 2 shows the resulting histogram of $x_{T}$ including 500 simulations.

In order to investigate the influence of the step-size, figure 3 illustrates four different histograms of 500 simulations. Here $h$ is $0.01,0.001,0.0001$ and 0.00001 respectively and $k=1$. It can be seen right away that the result narrows slightly around zero when $h$ becomes smaller, but changing in the step-size does not immediately give big effect.

In figure 4, the control gain is changing, $k=1,2,3,4$ and $h=0.001$. Here it is clear that changing $k$ has an influence on the result of $x_{T}$. The variance of $x_{T}$ gets smaller when $k$ increases. This is not surprising since the overall influence of the random variable $W_{n}$ is decreased when $k$ increases as mentioned in previous section.

\subsection{Theoretical Distribution of $x_{n}$}

In the following, we investigate a distribution of $x_{n}$ defined by the Euler-Maruyama method.

Consider the recursive determination of $x_{n+1}$ in (6). Define an intermediate variable $z_{n}=x_{n}+$ $h k \operatorname{sign}\left(x_{n}\right)$ and let $f_{z_{n}}$ and $f_{n}$ denote the density functions of $z_{n}$ and $x_{n}$, respectively. Moreover, let $N(0, h)$ denote the density function for $W_{n}$. From probability theory we get

$$
f_{n+1}(x)=f_{z_{n}} * N(0, h)
$$

where $*$ denotes convolution. 

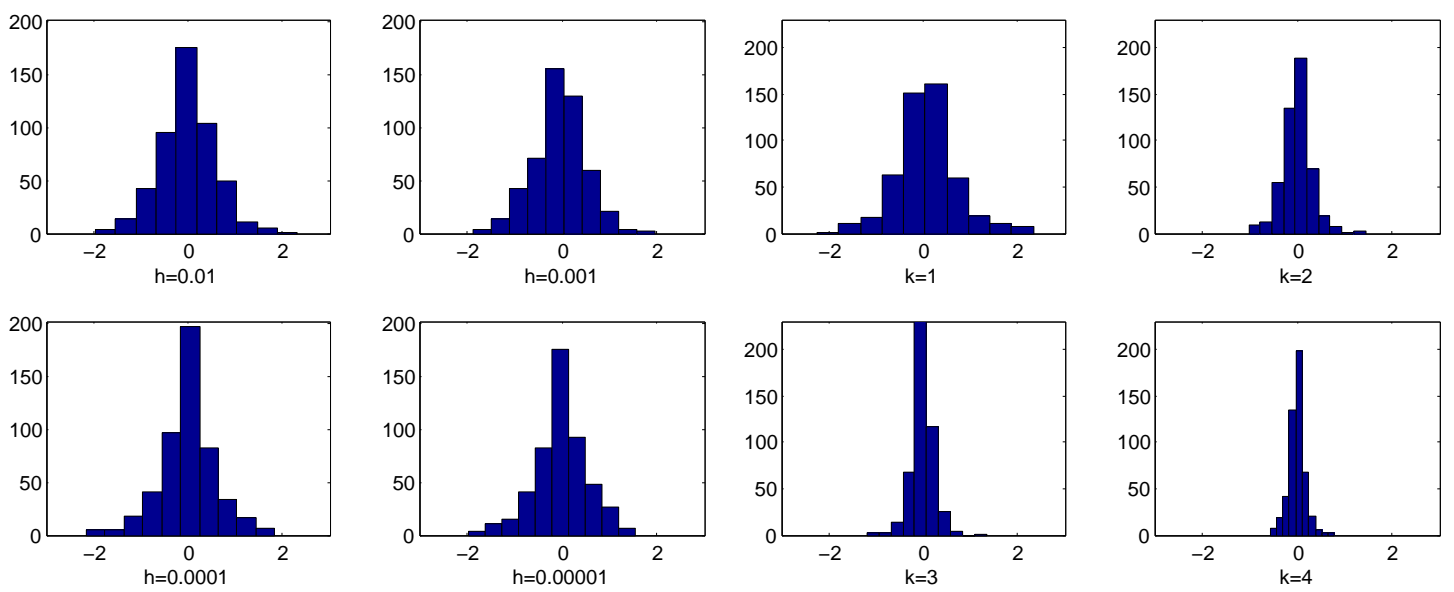

Figure 3: Histograms of 500 simulations of $x_{T}$ with different step-size.

Figure 4: Histograms of 500 simulations of $x_{T}$ with different control gains. $h=0.001$.

Hence, we proceed by studying the density function $f_{z_{n}}(z)$. Let the distribution function of $z_{n}$ be denoted by $F_{z_{n}}$ such that $F_{z_{n}}(z)=P\left(z_{n} \leq z\right)=P\left(x_{n}-h k \operatorname{sign}\left(x_{n}\right) \leq z\right)$, which can be expressed by

$$
\begin{aligned}
P\left(z_{n} \leq z\right) & =P\left(x_{n}-h k \leq z, x_{n}>0\right)+P\left(x_{n}+h k \leq z, x_{n}<0\right)+P\left(x_{n} \leq z, x_{n}=0\right) \\
& =P\left(x_{n} \leq z+h k, x_{n}>0\right)+P\left(x_{n} \leq z-h k, x_{n}<0\right) .
\end{aligned}
$$

For different values of $z$, the probability $P\left(z_{n} \leq z\right)$ can be expressed differently. If $z<-h k$

$$
P\left(z_{n} \leq z\right)=P\left(x_{n} \leq z-h k, x_{n}<0\right)=P\left(x_{n} \leq z-h k\right)=F_{n}(z-h k) .
$$

If $z>h k$

$$
P\left(z_{n} \leq z\right)=P\left(x_{n} \leq z+h k, x_{n}>0\right)+P\left(x_{n} \leq z-h k\right)=P\left(x_{n} \leq z+h k\right)=F_{n}(z+h k),
$$

and if $-h k \leq z \leq h k$

$$
\begin{aligned}
P\left(z_{n} \leq z\right) & =P\left(x_{n} \leq z+h k\right)-P\left(x_{n}<0\right)+P\left(x_{n} \leq z-h k\right) \\
& =F_{n}(z+h k)-F_{n}(0)+F_{n}(z-h k) .
\end{aligned}
$$

By introducing the indicator function $\mathbb{I}$, the expression of the distribution function of $z_{n}$ is

$$
\begin{aligned}
P\left(z_{n} \leq z\right) & =F_{n}(z-h k) \mathbb{I}_{(-\infty,-h k)}(z)+\left(F_{n}(z+h k)-F_{n}(0)+F_{n}(z-h k)\right) \mathbb{I}_{[-h k, h k]}(z)+F_{n}(z+h k) \mathbb{I}_{(h k, \infty)}(z) \\
& =F_{n}(z-h k) \mathbb{I}_{(-\infty, h k]}(z)+F_{n}(z+h k) \mathbb{I}_{[-h k, \infty)}(z)-F_{n}(0) \mathbb{I}_{[-h k, h k]}(z) .
\end{aligned}
$$

By differentiating with respect to $z$, the density function of $z_{n}$ is

$$
\begin{aligned}
\frac{\partial}{\partial z} F_{z_{n}}= & f_{n}(z-h k) \mathbb{I}_{(-\infty, h k]}(z)-F_{n}(z-h k) \delta(z-h k)+f_{n}(z+h k) \mathbb{I}_{[-h k, \infty)}(z) \\
& +F_{n}(z+h k) \delta(z+h k)-F_{n}(0)(\delta(z+h k)-\delta(z-h k)) \\
= & f_{n}(z-h k) \mathbb{I}_{(-\infty, h k]}(z)+f_{n}(z+h k) \mathbb{I}_{(-h k, \infty)}(z)+\delta(z+h k)\left(F_{n}(z+h k)-F_{n}(0)\right) \\
& +\delta(z-h k)\left(F_{n}(0)-F_{n}(z-h k)\right) \\
= & f_{n}(z-h k) \mathbb{I}_{(-\infty, h k]}(z)+f_{n}(z+h k) \mathbb{I}_{[-h k, \infty)}(z) .
\end{aligned}
$$




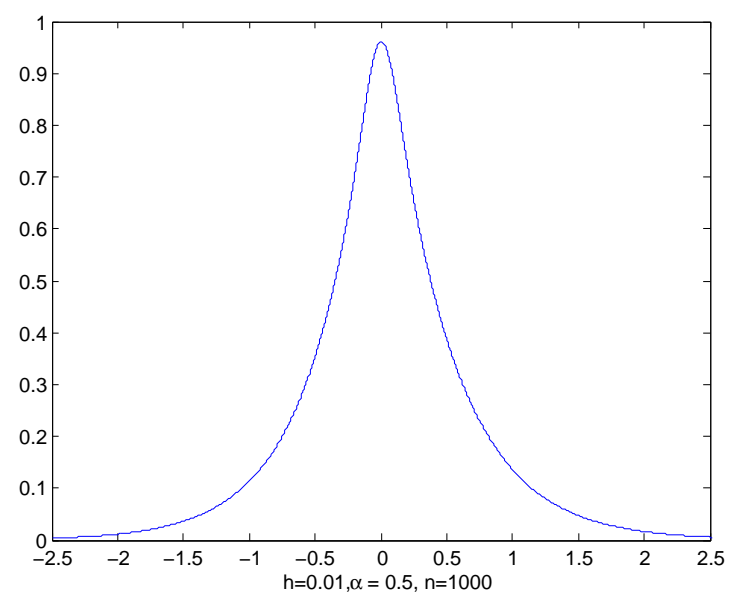

Figure 5: Recursive density function, $f_{1000}$.

Therefore

$$
f_{z_{n}}(z)=f_{n}(z-h k) \mathbb{I}_{(-\infty, h k]}(z)+f_{n}(z+h k) \mathbb{I}_{[-h k, \infty)}(z) .
$$

By substituting the above into (7), the density function $f_{n+1}$ is found from the density function $f_{n}$. In the following section, the solution to $(8)$ is investigated numerically.

\subsection{Recursive Developing of the Density Function in Matlab}

The recursive density function is given by

$$
f_{n+1}(x)=\left(f_{n}(x+k h) \mathbb{I}_{(-\infty, h k]}+f_{n}(x-h k) \mathbb{I}_{[-h k, \infty)}\right) * N(0, h) .
$$

Following, we apply Matlab to investigate the evolution of the function $f_{n+1}(x)$ for $n$ increasing. Assume that the density function for the initial condition $x_{0}=c$ is normal distributed with mean zero and variance $h$. The Euler-Maruyama method is expected to converge to a stochastic process (or a distribution of a stochastic process) when $h \rightarrow 0$. (Under certain regularity conditions, so actually we cannot expect it here but only conjecture.) We hope that the developing of the recursive density functions in (9) will reach stationary condition for $n \rightarrow \infty$. For this reason, the number $n$ of simulations is chosen to depend on the step size, such that $n=\left\lceil\frac{1}{h^{1+\alpha}}\right\rceil$, where $\alpha>0$. This ensures that both convergence criteria are fulfilled.

Equation (9) is simulated in Matlab for $h=0.01, \alpha=0.5, k=1$ such that $n=1000$, the result is shown in figure 5. At the end of section 3, a comparison between the convergence of the recursive density function and the result obtained there is presented.

In the following, we continue the study of $(8)$ under stationary assumptions.

\subsection{Stationary State}

In the sequel we continue the study of the recursive density function under the assumptions that it is possible to reach stationary state in $(9)$ for $n \rightarrow \infty$, such that

$$
f_{z_{n}}(z)=f(z-h k) \mathbb{I}_{(-\infty, h k]}(z)+f(z+h k) \mathbb{I}_{(-h k, \infty)}(z),
$$


where $f(x)$ is the stationary density function of the recursive development of $f_{n}(x)$. In this case we define an operator $H_{h}$ taking $f_{n}$ to $f_{n+1}$ by

$$
H_{h}[f](x)=\int_{\mathbb{R}}\left(f(z-h k) \mathbb{I}_{(-\infty, h k]}(z)+f(z+h k) \mathbb{I}_{(-h k, \infty)}(z)\right) \frac{1}{\sqrt{2 \pi h}} \exp \left(\frac{-(x-z)^{2}}{2 h}\right) d z .
$$

By taking the derivative with respect to $h$ and then the limit $h \rightarrow 0$, we obtain (see appendix A.1),

$$
\lim _{h \rightarrow 0} \frac{\partial}{\partial h} H_{h}[f](x)=\left\{\begin{array}{ccc}
-k f^{\prime}(x)+\frac{1}{2} f^{\prime \prime}(x) & \text { for } & x<0 \\
\psi(x) & \text { for } & x=0 \\
k f^{\prime}(x)+\frac{1}{2} f^{\prime \prime}(x) & \text { for } & x>0
\end{array} .\right.
$$

The case $x=0$ is not important for the sequel developing, hence we leave $\psi(x)$ unspecified. Note that the right hand side of the first and last case in 10 have similarity with the stationary Fokker-Planck equation.

An approximation of the operator $H_{h}$ is

$$
\begin{aligned}
H_{h}[f](x) & \approx f(x)+h\left(k \operatorname{sign}(x) f^{\prime}(x)+\frac{1}{2} f^{\prime \prime}(x)\right) \\
& =f(x)+h G[f](x) .
\end{aligned}
$$

For a fixed $h$ define $f_{h}$ to be the stationary density function and assume that $\lim _{h \rightarrow 0} f_{h}$ exists, say $f_{0}$ and that $H_{h}\left(f_{h}\right)=f_{h}$. Hence, if we disregard the approximation in (11), we look for a function $f_{h}$ such that $G\left[f_{h}\right]=0$, which by $(10)$ means that $f_{h}$ is a stationary solution to the Fokker-Planck equation. We conjecture that this heuristic will constitute the main ideas in the proof that the stationary distribution $f_{0}\left(=\lim _{h \rightarrow 0} f_{h}\right)$ of the Euler-Maruyama simulation converges to the Fokker-Planck equation.

\section{Fokker-Planck Equation}

Based on the preceding work, this section introduces the one-dimensional Fokker-Planck equation and apply this to determine a density function of $x_{t}$ for fixed $t$.

Let $x_{t}$ be a solution to (4). For a fixed $t \in[0, T]$ let $\phi(x, t)$ be the density function of $x_{t}$, such that

$$
\int_{\mathbb{R}} \phi(x, t) d x=1
$$

with initial condition

$$
\lim _{t \rightarrow 0} \phi(x, t)=\delta\left(x-x_{0}\right),
$$

where $\delta$ denotes the Dirac-delta function. If the drift and diffusion coefficient are moderately smooth functions, then the density function $\phi(x, t)$ satisfies the Fokker-Plank equation [16, 1]. For (4) this means

$$
\frac{\partial}{\partial t} \phi(x, t)=\frac{\partial}{\partial x} k \operatorname{sign}(x) \phi(x, t)+\frac{1}{2} \frac{\partial^{2}}{\partial x^{2}} \phi(x, t) .
$$

As mentioned previous, the drift coefficient $-k \operatorname{sign}(x)$ is not a smooth function and for this reason there is no guarantee that (13) is valid. 


\subsection{Solution in Two Domains}

In order to avoid the discontinuous challenges by the sign-function, we consider the Fokker-Planck equation (13) in the domains $(-\infty, 0)$ and $(0, \infty)$. This gives

$$
\begin{aligned}
\frac{\partial}{\partial t} \phi(x, t) & =\frac{\partial}{\partial x} k \phi(x, t)+\frac{1}{2} \frac{\partial^{2}}{\partial x^{2}} \phi(x, t) \text { for } x>0 \\
\frac{\partial}{\partial t} \phi(x, t) & =-\frac{\partial}{\partial x} k \phi(x, t)+\frac{1}{2} \frac{\partial^{2}}{\partial x^{2}} \phi(x, t) \text { for } x<0 .
\end{aligned}
$$

Assume that the density function can reach stationarity, then

$$
\begin{aligned}
0 & =\frac{\partial}{\partial x} k \phi(x, t)+\frac{1}{2} \frac{\partial^{2}}{\partial x^{2}} \phi(x, t) \text { for } x>0 \\
0 & =-\frac{\partial}{\partial x} k \phi(x, t)+\frac{1}{2} \frac{\partial^{2}}{\partial x^{2}} \phi(x, t) \text { for } x<0,
\end{aligned}
$$

which are two ODEs. The characteristic equations are

$$
\begin{aligned}
k s+\frac{1}{2} s^{2} & =0 \text { for } x>0 \\
-k s+\frac{1}{2} s^{2} & =0 \text { for } x<0,
\end{aligned}
$$

which have the roots

$$
\begin{aligned}
& s=0, \quad s=-2 k \quad \text { for } x>0 \\
& s=0, \quad s=2 k \quad \text { for } x<0,
\end{aligned}
$$

such that

$$
\begin{aligned}
& \phi_{+}(x)=c_{1} \exp (0 x)+d_{1} \exp (-2 k x) \text { for } x>0 \\
& \phi_{-}(x)=c_{2} \exp (0 x)+d_{2} \exp (2 k x) \text { for } x<0
\end{aligned}
$$

are solutions of (13) in the respective domain. We discard the constant term and, due to symmetry around $x=0$, it can be expected that $d_{1}=d_{2}:=d$. The boundary constraint in equation (12) gives

$$
d=\frac{1}{2 \int_{0}^{\infty} \exp (-2 k x)}=k
$$

Furthermore, from the above we assume that $\phi(x, t)=k$ for $x=0$. This gives the density function

$$
\phi(x, t)=\left\{\begin{array}{cc}
k \exp (-2 k x) & \text { for } x>0 \\
k \exp (2 k x) & \text { for } x<0 \\
k & \text { for } x=0
\end{array}\right.
$$

which is illustrated in figure 6 for $k=1$. The density function $\phi(x, t)$ is the Laplace distribution with location parameter zero and scale parameter $\frac{1}{2 k}$.

The density function in (14) is compared with the developing of the recursive density functions from section 2.4 by considering the logarithmic of the density functions. The recursive cases with $n=$ $158,398,1000$ are shown in figure 7 together with the logarithmic stationary density function $\log (\phi(x, t))$. For increasing $n$, the logarithmic recursive density function gets closer to the logarithmic stationary density function, as we hope to observe.

Due to the lack of continuity in all the previous investigations, in the following section we construct a smooth function which approximate the sign-function and investigate which results the applied methods provide with this smooth function as input. 


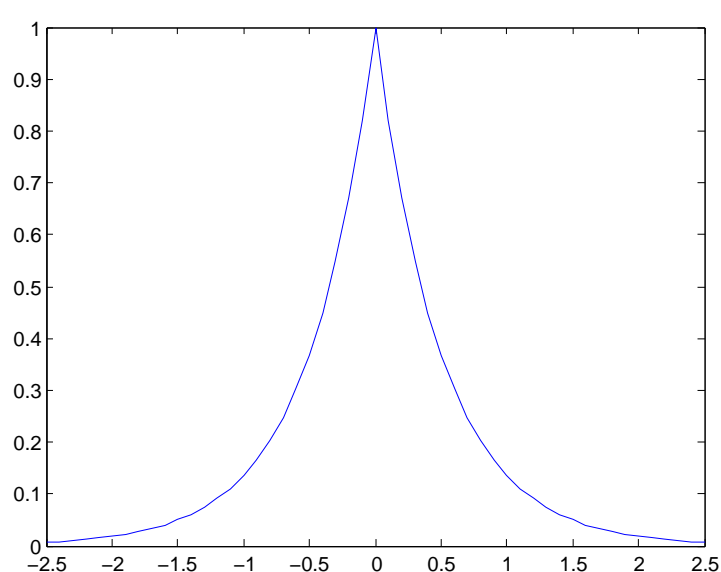

Figure 6: Density function $\phi(x, t)$ for $x_{t}$ for fixed $t \in[0, T]$.

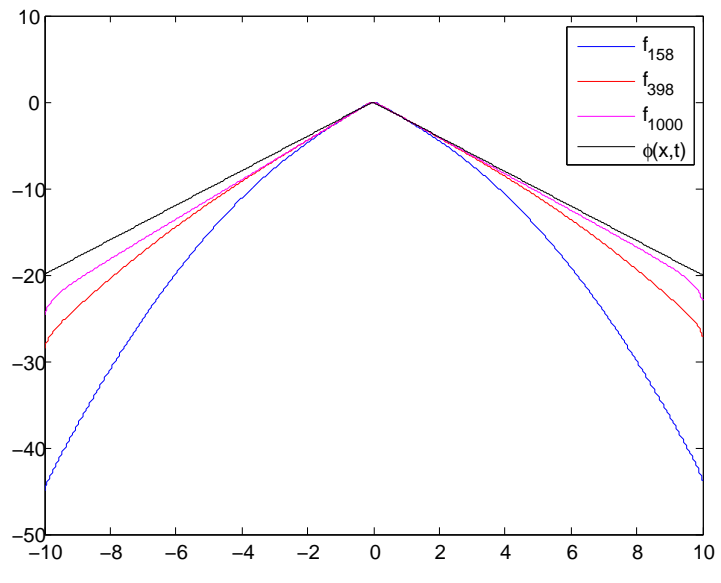

Figure 7: Logarithmic stationary density function and logarithmic recursive density function of $f_{158}, f_{398}, f_{1000}$.

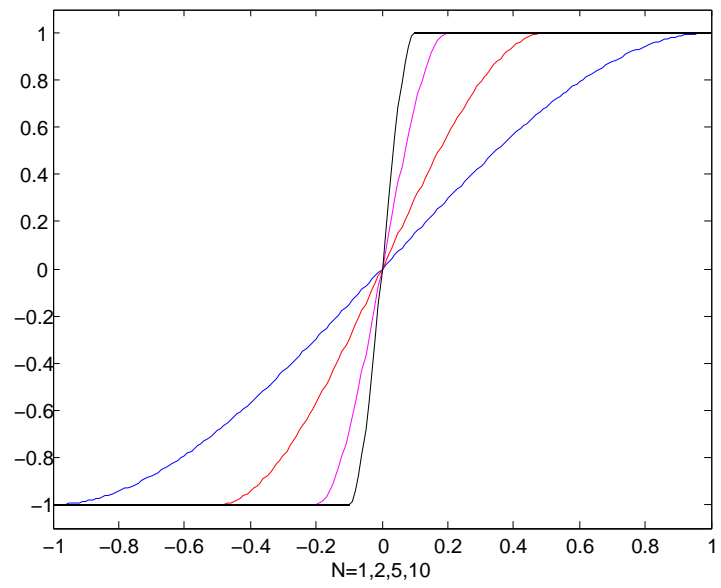

Figure 8: Approximation of the sign-function.

\section{Approximation of sign-Function}

As mentioned, the problem with the sign-function is that it is not smooth. For this reason we construct a function $f_{N}(x)$ which has pointwise convergence to the sign-function as $N \rightarrow \infty$.

$$
f_{N}(x)=\left\{\begin{array}{cl}
1 & \text { for } x>\frac{1}{N} \\
-\frac{N^{3}}{2} x^{3}+\frac{3 N}{2} x & \text { for }-\frac{1}{N} \leq x \leq \frac{1}{N} \\
-1 & \text { for } x<-\frac{1}{N}
\end{array}\right.
$$

In figure 8 , the functions $f_{1}(x), f_{2}(x), f_{5}(x)$ and $f_{10}(x)$ are shown. In the following, we will apply the Euler-Maruyama method to this function. 


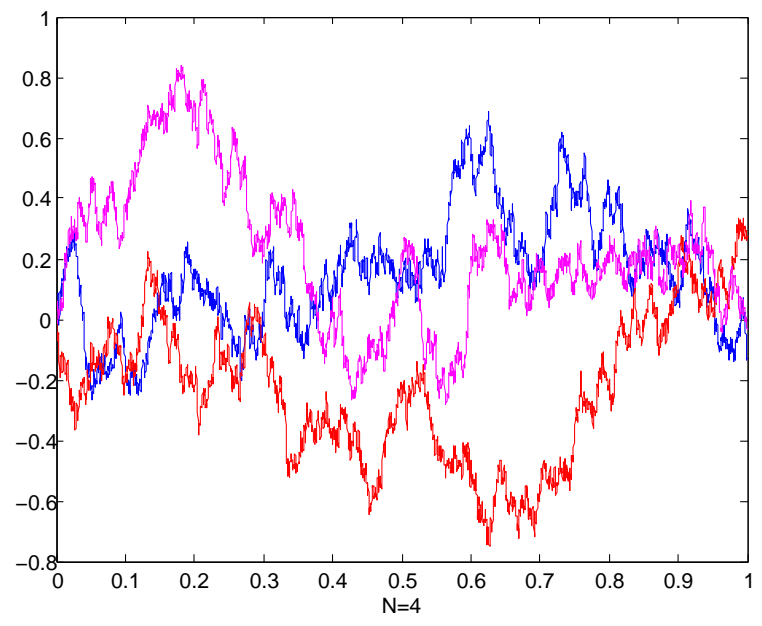

Figure 9: Realizations of $x_{t}$ to a SDE with drift $b\left(t, x_{t}\right)=-k f_{4}\left(x_{t}\right)$.
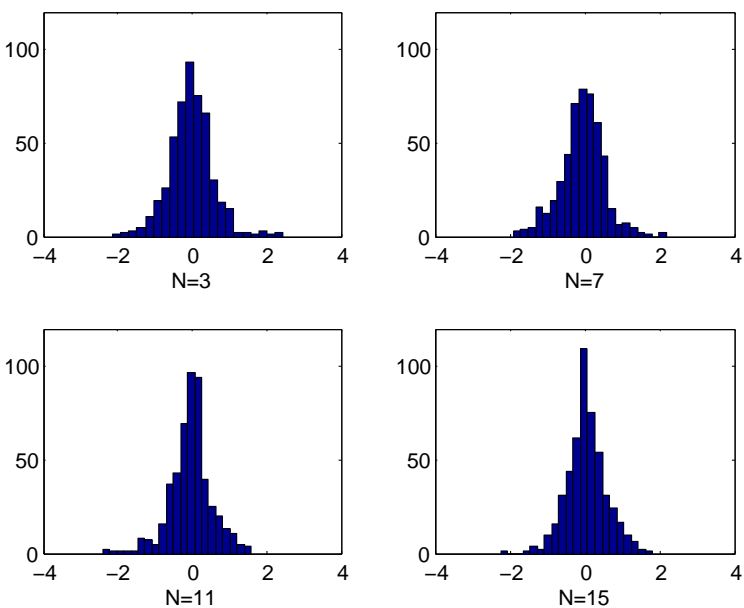

Figure 10: Histogram of 500 simulations of $x_{T}$ where different approximations to the sign-function are used.

\subsection{Euler-Maruyama Method Applied to the Approximated sign-Function}

It is proved that the Euler-Maruyama method exhibits convergence to the solution of the SDE if the drift and diffusion coefficient satisfy certain regularity conditions. Since $f_{N}(x)$ from (15) is a smooth function, we apply the Euler-Maruyama method with this input instead of the sign-function. The same procedure as in section 2.2 is applied, such that

$$
x_{j+1}=x_{j}-h k f_{N}\left(x_{j}\right)+W_{j} .
$$

Since the slope of $f_{N}(x)$ increases significantly with the increase of $N$, the step-size is chosen to be dependent on $N$, such that $h=\frac{0.001}{N}$. We hope to avoid inaccurate simulations due to the step-size with this method. (If $x_{i} \in\left[-\frac{1}{N}, \frac{1}{N}\right]$ for $t_{i} \in[0, T]$ and $N$ is big, then the value of $f_{N}\left(x_{i}\right)$ is dominating in the determination of $x_{i+1}$ such that $x_{i}<<x_{i+1}$ or $x_{i}>>x_{i+1}$.) As previous, $k=1$. In figure 9 , are shown 3 realizations of $x_{t}$ when $f_{4}(x)$ is used to approximate $\operatorname{sign}(x)$. In figure 10 histograms of 500 simulations are presented based on different approximations to the sign-function.

In the following section, the smooth function is implemented in the Fokker-Planck equation.

\subsection{Solution to the Fokker-Planck Equation with Approximated sign-function}

Previous, the Fokker-Planck equation have been applied to a SDE where the drift is discontinuous due to the sign-function. As mentioned, we cannot expect this to be meaningful. Following the smooth function $f_{N}(x)$ is used as a substitute for the sign-function, such that we consider the SDE

$$
d x_{t}=-k f_{N}\left(x_{t}\right) d t+d B_{t} .
$$

We investigate if it is possible to determine a density function that is a solution to the Fokker-Planck equation with $-k f_{N}(x)$ as the drift.

With same technique as previous we consider the Fokker-Planck equation in three domains. First the domain $\left[-\frac{1}{N}, \frac{1}{N}\right]$ is considered with $f_{N}(x)$ as an approximation of $\operatorname{sign}(x)$.

$$
\frac{\partial}{\partial t} \phi_{N}(x, t)=\frac{\partial}{\partial x} k\left(-\frac{N^{3}}{2} x^{3}+\frac{3 N}{2} x\right) \phi_{N}(x, t)+\frac{1}{2} \frac{\partial^{2}}{\partial x^{2}} \phi_{N}(x, t) .
$$


Under stationary assumptions, the function $\phi_{N}(x, t)=\phi_{0} \exp \left(\frac{N^{3}}{4} k x^{4}-\frac{3 N}{2} k x^{2}\right)$ fulfills 18 ). By including the domains $\left(-\infty,-\frac{1}{N}\right)$ and $\left(\frac{1}{N}, \infty\right)$, the density function for solutions to (17) becomes

$$
\phi_{N}(x, t)=\left\{\begin{array}{cl}
d \exp (2 k x) & \text { for } x<-\frac{1}{N} \\
\phi_{0} \exp \left(\frac{N^{3}}{4} k x^{4}-\frac{3 N}{2} k x^{2}\right) & \text { for }-\frac{1}{N} \leq x \leq \frac{1}{N} \\
d \exp (-2 k x) & \text { for } x>\frac{1}{N}
\end{array},\right.
$$

with constraints

$$
1=\int_{-\infty}^{-\frac{1}{N}} d \exp (2 k x) d x+\int_{\frac{1}{N}}^{\infty} d \exp (-2 k x) d x+\int_{-\frac{1}{N}}^{\frac{1}{N}} \phi_{0} \exp \left(k\left(\frac{N^{3}}{4} x^{4}-\frac{3 N}{2} x^{2}\right)\right) d x
$$

and

$$
\lim _{x \rightarrow \pm \frac{1}{N}+} \phi_{N}(x, t)=\lim _{x \rightarrow \pm \frac{1}{N}-} \phi_{N}(x, t) .
$$

From (19)

$$
d=\phi_{0} \exp \left(\frac{3 k}{4 N}\right)
$$

so the normalization constant becomes

$$
\phi_{0}=\frac{1}{2 \int_{0}^{\frac{1}{N}} \exp \left(-\frac{3 k N}{2} x^{2}+\frac{k N^{3}}{4} x^{4}\right) d x+\frac{1}{k} \exp \left(-\frac{5 k}{4 N}\right)}
$$

and

$$
\phi_{N}(x, t)=\left\{\begin{array}{cl}
\phi_{0} \exp \left(\frac{3 k}{4 N}\right) \exp (2 k x) & \text { for } x<-\frac{1}{N} \\
\phi_{0} \exp \left(\frac{N^{3}}{4} k x^{4}-\frac{3 N}{2} k x^{2}\right) & \text { for }-\frac{1}{N} \leq x \leq \frac{1}{N} \\
\phi_{0} \exp \left(\frac{3 k}{4 N}\right) \exp (-2 k x) & \text { for } x>\frac{1}{N}
\end{array} .\right.
$$

This density function is simulated in Matlab with $\phi_{0}$ numerically calculated. In figure 11 results are shown for $N=1,10,100,1000,10000$ together with the stationary density function from the FokkerPlanck equation $\phi(x, t)$. Note that $\phi_{100}, \phi_{1000}$ and $\phi_{10000}$ are not visible. The simulations show that $\phi_{N}(x, t) \rightarrow \phi(x, t)$ as expected.

\section{Fourier Transformation}

In section 2.3 we investigate a candidate density function for the intermediate variable $z_{n}$ in order to determine a density function for the stochastic process produced by Euler-Maruyama method. Another method than the one presented there is to Fourier transform the density function for $z_{n}$ before the convolution of the density functions is done. The Fourier transformation of 8 ) is given by

$$
\hat{F}(\omega)=\int_{\mathbb{R}}\left[f_{n}(z-h k) \mathbb{I}_{(-\infty, h k]}+f_{n}(z+h k) \mathbb{I}_{(-h k, \infty)}\right] \exp (-j \omega z) d z
$$

By expansion and including of the Laplace transformation, see appendix A.2, the following relation appears:

$$
\hat{F}(\omega)=\cos (\omega h k) \operatorname{Re}(\hat{F}(\omega))-2 \sin (\omega h k) \operatorname{Im}(\tilde{F}(\omega)),
$$

where $\tilde{F}(\omega)$ is the Laplace transformation. For the moment we have no further interpretation of how this can help the developing of the recursive density function. 


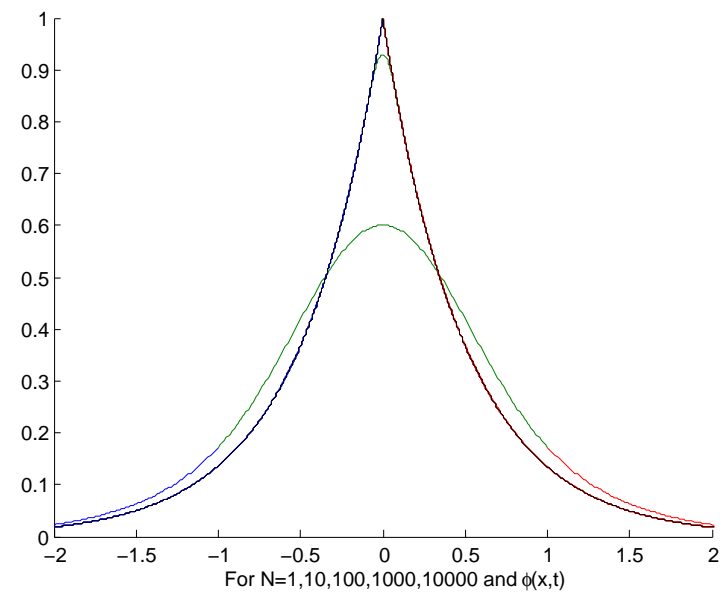

Figure 11: Density functions for solutions to (17) together with $\phi(x, t)$.

\section{Discussion}

This paper has presented initial studies of SDEs with discontinuous drift. Theoretical and numerical approaches have been applied to a particular SDE in order to investigate meaningful results in the form of density functions.

The candidate recursive density function developed from the Euler-Maruyama method has the tendency to approximate the density function of the stationary Fokker-Planck equation, which strengthens the conjecture that these density functions actually exist. Furthermore, the fact that the Laplace distribution is a solution to the stationary Fokker-Planck equation with discontinuous drift supports the assumption that it is possible to give a meaningful answering to questions about definition of solutions to discontinuous SDEs and their probabilistic properties. However, we have not completed these processes, yet.

All in all, the different approaches indicate a connection between the candidate density function for the solution to the particular SDE with discontinuous drift and the Fokker-Planck equation. Though, the stationary assumptions have extensive impact on this result. Without this condition, the solvability of the Fokker-Planck equation would decrease significantly.

An immediate object for future studies is to formalize the heuristic presentation in this paper to obtain operational definition and regular results connected to classes of SDEs with discontinuous drift.

\section{A Appendix}

\section{A.1 Approximation of the operator $H_{h}$}

In section 2.5 the operator $H_{h}$ was defined by

$$
H_{h}[f](x)=\int_{\mathbb{R}}\left(f(z-h k) \mathbb{I}_{(-\infty, h k]}(z)+f(z+h k) \mathbb{I}_{(-h k, \infty)}(z)\right) \frac{1}{\sqrt{2 \pi h}} \exp \left(\frac{-(x-z)^{2}}{2 h}\right) d z,
$$


Following, a notation $N_{h}(z)=\frac{1}{\sqrt{2 \pi h}} \exp \left(\frac{-z^{2}}{2 h}\right)$ is used and we consider the generator $\frac{\partial}{\partial h} H_{h}$.

$$
\begin{aligned}
\frac{\partial}{\partial h} H_{h}[f](x)= & \int_{\mathbb{R}}\left(-k f^{\prime}(z-h k) \mathbb{I}_{(-\infty, h k]}(z)+k f^{\prime}(z+h k) \mathbb{I}_{[-h k, \infty)}(z)\right) \cdot N_{h}(x-z) d z \\
& +\int_{\mathbb{R}}(-k f(z-h k) \delta(z-h k)+k f(z+h k) \delta(z+h k)) \cdot N_{h}(x-z) d z \\
& +\int_{\mathbb{R}}\left(f(z-h k) \mathbb{I}_{(-\infty, h k]}(z)+f(z+h k) \mathbb{I}_{[-h k, \infty)}(z)\right) \frac{\partial}{\partial h}\left(N_{h}(x-z)\right) d z .
\end{aligned}
$$

The second integral term above gives

$$
\begin{aligned}
& \int_{\mathbb{R}}(-k f(z-h k) \delta(z-h k)+k f(z+h k) \delta(z+h k)) \cdot N_{h}(x-z) d z \\
= & k f(0)\left(N_{h}(x+h k)-N_{h}(x-h k)\right) .
\end{aligned}
$$

The derivative of the last integral term in 20 gives

$$
\frac{\partial}{\partial h}\left(N_{h}(x-z)\right)=\frac{1}{\sqrt{2 \pi h}}\left(\frac{(x-z)^{2}}{2 h^{2}}-\frac{1}{2 h}\right) \exp \left(\frac{-(x-z)^{2}}{2 h}\right) .
$$

Consider the derivative with respect to $z$,

$$
\frac{\partial}{\partial z}\left(N_{h}(x-z)\right)=\frac{1}{\sqrt{2 \pi h}} \frac{-(x-z)}{h} \exp \left(\frac{-(x-z)^{2}}{2 h}\right),
$$

and the second derivative with respect to $z$,

$$
\frac{\partial^{2}}{\partial z^{2}}\left(N_{h}(x-z)\right)=\frac{1}{\sqrt{2 \pi h}}\left(\frac{(x-z)^{2}}{h^{2}}+\frac{1}{h}\right) \exp \left(\frac{-(x-z)^{2}}{2 h}\right) .
$$

Thus, for small $h$

$$
\frac{\partial}{\partial h}\left(N_{h}(x-z)\right) \approx \frac{1}{2} \frac{\partial^{2}}{\partial z^{2}}\left(N_{h}(x-z)\right)
$$

Therefore, the last integral term in $\frac{\partial}{\partial h} H_{h}[f](x)$ is approximately

$$
\begin{aligned}
& \frac{1}{2} \int_{\mathbb{R}}\left(f(z-h k) \mathbb{I}_{(-\infty, h k]}(z)+f(z+h k) \mathbb{I}_{[-h k, \infty)}(z)\right) \frac{\partial^{2}}{\partial z^{2}}\left(N_{h}(x-z)\right) d z \\
= & \frac{1}{2} \int_{-\infty}^{h k} f(z-h k) \frac{\partial^{2}}{\partial z^{2}}\left(N_{h}(x-z)\right) d z+\frac{1}{2} \int_{-h k}^{\infty} f(z+h k) \frac{\partial^{2}}{\partial z^{2}}\left(N_{h}(x-z)\right) d z \\
= & \frac{1}{2}\left(\left[f(z-h k) \frac{\partial}{\partial z}\left(N_{h}(x-z)\right)\right]_{-\infty}^{h k}-\left[f^{\prime}(z-h k) N_{h}(x-z)\right]_{-\infty}^{h k}+\int_{-\infty}^{h k} f^{\prime \prime}(z-h k) N_{h}(x-z) d z\right) \\
& +\frac{1}{2}\left(\left[f(z+h k) \frac{\partial}{\partial z}\left(N_{h}(x-z)\right)\right]_{-h k}^{\infty}-\left[f^{\prime}(z+h k) N_{h}(x-z)\right]_{-h k}^{\infty}+\int_{-h k}^{\infty} f^{\prime \prime}(z+h k) N_{h}(x-z) d z\right) .
\end{aligned}
$$

The limit of $\frac{\partial}{\partial h} H_{h}$ for $h \rightarrow 0$ is then

$$
\lim _{h \rightarrow 0} \frac{\partial}{\partial h} H_{h}[f](x)=\left\{\begin{array}{ll}
-k f^{\prime}(x)+\frac{1}{2} f^{\prime \prime}(x) & \text { for } \quad x<0 \\
\psi(x) & \text { for } \quad x=0 \\
k f^{\prime}(x)+\frac{1}{2} f^{\prime \prime}(x) & \text { for } \quad x>0
\end{array},\right.
$$

where $\psi(x)$ is unknown. 


\section{A.2 Fourier Transformation Expansion}

In section 5 the Fourier transformation of $f_{z_{n}}$ is presented. Below follows the expansion.

$$
\begin{aligned}
\hat{F}(\omega) & =\int_{\mathbb{R}}\left[f_{n}(z-h k) \mathbb{I}_{(-\infty, h k]}+f_{n}(z+h k) \mathbb{I}_{(-h k, \infty)}\right] \exp (-j \omega z) d z \\
& =\int_{-\infty}^{h k} f_{n}(z-h k) \exp (-j \omega z) d z+\int_{-h k}^{\infty} f_{n}(z+h k) \exp (-j \omega z) d z .
\end{aligned}
$$

By changing variable $x=z-h k$ in the first integral and $x=z+h k$ in the second integral,

$$
\begin{aligned}
\hat{F}(\omega)= & \int_{-\infty}^{0} f_{n}(x) \exp (-j \omega(x+h k)) d x+\int_{0}^{\infty} f_{n}(x) \exp (-j \omega(x-h k)) d x \\
= & \exp (-j \omega h k)) \int_{-\infty}^{0} f_{n}(x) \exp (-j \omega x) d x+\exp (j \omega h k) \int_{0}^{\infty} f_{n}(x) \exp (-j \omega x) d x \\
= & (\cos (\omega h k)-j \sin (\omega h k)) \int_{-\infty}^{0} f_{n}(x) \exp (-j \omega x) d x \\
& \quad+(\cos (\omega h k)+j \sin (\omega h k)) \int_{0}^{\infty} f_{n}(x) \exp (-j \omega x) d x .
\end{aligned}
$$

Based on the uncertainty around zero in the recursive determination of $x_{n+1}$ in (5), we find it fair to assume that $f_{n}$ is an even function. Fourier transformations of even functions give zero in the imaginary part so only the real part of (21) is considered.

$$
\begin{aligned}
\hat{F}(\omega)= & \cos (\omega h k) \operatorname{Re}\left(\int_{-\infty}^{0} f_{n}(x) \exp (-j \omega x) d x\right)+\sin (\omega h k) \operatorname{Im}\left(\int_{-\infty}^{0} f_{n}(x) \exp (-j \omega x) d x\right) \\
& +\cos (\omega h k) \operatorname{Re}\left(\int_{0}^{\infty} f_{n}(x) \exp (-j \omega x) d x\right)-\sin (\omega h k) \operatorname{Im}\left(\int_{0}^{\infty} f_{n}(x) \exp (-j \omega x) d x\right) \\
= & \cos (\omega h k) \int_{-\infty}^{0} f_{n}(x) \cos (\omega x) d x-\sin (\omega h k) \int_{-\infty}^{0} f_{n}(x) \sin (\omega x) d x \\
& +\cos (\omega h k) \int_{0}^{\infty} f_{n}(x) \cos (\omega x) d x+\sin (\omega h k) \int_{0}^{\infty} f_{n}(x) \sin (\omega x) d x \\
= & \cos (\omega h k) \int_{-\infty}^{\infty} f_{n}(x) \cos (\omega x) d x+2 \sin (\omega h k) \int_{0}^{\infty} f_{n}(x) \sin (\omega x) d x \\
= & \cos (\omega h k) \operatorname{Re}(\hat{F}(\omega))+2 \sin (\omega h k) \int_{0}^{\infty} f_{n}(x) \sin (\omega x) d x
\end{aligned}
$$

Consider the Laplace transformation of $f_{n}(x)$,

$$
\tilde{F}(s)=\mathscr{L}\left\{f_{n}(x)\right\}=\int_{0}^{\infty} f_{n}(x) \exp (-s x) d x .
$$

The imaginary part of 23 is

$$
\operatorname{Im}(\tilde{F}(s))=-\int_{0}^{\infty} f_{n}(x) \sin (s x) d x .
$$

From this 22, can be expressed by

$$
\hat{F}(\omega)=\cos (\omega h k) \operatorname{Re}(\hat{F}(\omega))-2 \sin (\omega h k) \operatorname{Im}(\tilde{F}(\omega)),
$$

where $\tilde{F}(\omega)$ is the Laplace transformation. 


\section{References}

[1] Ludwig Arnold (1974): Stochastic differential equations: theory and applications. Wiley-Interscience [John Wiley \& Sons], New York. Translated from the German.

[2] Manuela L. Bujorianu \& John Lygeros (2006): Toward a general theory of stochastic hybrid systems. In: Stochastic hybrid systems, Lecture Notes in Control and Inform. Sci. 337, Springer, Berlin, pp. 3-30, doi:10.1007/11587392_1.

[3] A Einstein (1905): Über die von der molekularkinetischen Theorie der Wärme geforderte Bewegung von in ruhenden Flüssigkeiten suspendierten Teilchen. Annalen der Physik 17(8), pp. 549-560, doi: $10.1002 /$ andp. 19053220806

[4] A. F. Filippov (1988): Differential equations with discontinuous righthand sides. Mathematics and its Applications (Soviet Series) 18, Kluwer Academic Publishers Group, Dordrecht. Translated from the Russian.

[5] Desmond J. Higham (2001): An algorithmic introduction to numerical simulation of stochastic differential equations. SIAM Rev. 43(3), pp. 525-546 (electronic), doi:10.1137/S0036144500378302.

[6] Jianghai Hu, John Lygeros \& Shankar Sastry (2000): Towards a Theory of Stochastic Hybrid Systems. N. Lynch and B. Krogh (Eds.): HSCC 2000, LNCS 1790, pp. 160-173.

[7] Kiyosi Ito (1951): On stochastic differential equations. Mem. Amer. Math. Soc. 1951(4), p. 51.

[8] Rafail Khasminskii (2012): Stochastic stability of differential equations, second edition. Stochastic Modelling and Applied Probability 66, Springer, Heidelberg, doi:10.1007/978-3-642-23280-0. With contributions by G. N. Milstein and M. B. Nevelson.

[9] Peter E. Kloeden \& Eckhard Platen (1992): Numerical solution of stochastic differential equations. Applications of Mathematics (New York) 23, Springer-Verlag, Berlin, doi 10.1007/978-3-662-12616-5

[10] Harold Kushner (1971): Introduction to stochastic control. Holt, Rinehart and Winston, Inc., New York.

[11] Harold J. Kushner (1967): Stochastic stability and control. Mathematics in Science and Engineering, Vol. 33, Academic Press, New York.

[12] C. Le Bris \& P.-L. Lions (2008): Existence and uniqueness of solutions to Fokker-Planck type equations with irregular coefficients. Comm. Partial Differential Equations 33(7-9), pp. 1272-1317, doi $10.1080 / 03605300801970952$

[13] Xuerong Mao \& Chenggui Yuan (2006): Stochastic differential equations with Markovian switching. Imperial College Press, London, doi:10.1142/p473.

[14] Gisirō Maruyama (1955): Continuous Markov processes and stochastic equations. Rend. Circ. Mat. Palermo (2) 4, pp. 48-90, doi:10.1007/BF02846028

[15] Bernt Øksendal (2003): Stochastic differential equations, sixth edition. Universitext, Springer-Verlag, Berlin, doi 10.1007/978-3-642-14394-6 An introduction with applications.

[16] H. Risken (1989): The Fokker-Planck equation, second edition. Springer Series in Synergetics 18, SpringerVerlag, Berlin, doi:10.1007/978-3-642-61544-3. Methods of solution and applications.

[17] R. L. Stratonovich (1966): A new representation for stochastic integrals and equations. SIAM J. Control 4, pp. 362-371, doi: $10.1137 / 0304028$ 